\title{
Hedgehogs in a Three-Dimensional Anisotropic Spin System
}

\author{
Thordur Jonsson \\ Nordita, Blegdamsvej 17, DK-2100 Copenhagen $\varnothing$, Denmark
}

\begin{abstract}
We study a continuum version of a classical anisotropic spin model in three dimensions with three component spins. We prove the existence of topological defects, called hedgehogs, which are analogous to the vortices in the two-dimensional $x y$-model and have a logarithmically divergent action. Bounds for the interaction energy of a hedgehog and an antihedgehog are derived.
\end{abstract}

\section{Introduction}

It has been known for some time now that topological defects can play an important role in the statistical mechanics of materials as well as in field theories. In this paper we study defects that arise in a classical continuum spin system (nonlinear $\sigma$-model) whose energy (action) is given by the anisotropic functional

$$
A(\mathbf{S})=\int\left\{\left(\partial_{z} \mathbf{S}\right)^{2}+(\Delta \mathbf{S})^{2}\right\} d^{3} x
$$

Here $\mathbf{S}$ is a three component spin vector of unit length, $x, y, z$ are Cartesian coordinates in $\mathbb{R}^{3}$ and $\Delta$ is the Laplacian in the $x y$-plane.

The action (1.1) comes up in the study of the Lifshitz point problem in magnetic systems [1]. The Lifshitz point is also of interest for phase transitions in liquid crystals and other systems, see $[2,3]$ and references therein. The case of two component spins has been analyzed in detail by Grinstein, Pelcovits, Nelson and Toner [3-6].

When the spin has three components we shall see that there exist pointlike topological defects that might strongly influence the thermodynamic behaviour of the system. This is the prime motivation for studying (1.1). The action of the defects is logarithmically divergent. The aim of this paper is to prove that there exist such defects and to estimate their interaction energy.

One could add a term proportional to $(\nabla \mathbf{S})^{4}$ to the functional(1.1), where $\nabla$ is the gradient in the $x y$-plane, without substantially changing our results. We choose to study (1.1) for the sake of simplicity. However, a term proportional to $(\nabla \mathbf{S})^{2}$ would cause a linear divergence in the action of the defects, see [7]. 
Let $\Sigma$ be a topological sphere in $\mathbb{R}^{3}$ enclosing the origin. Let

$$
\mathbf{S}: \mathbb{R}^{3} \backslash\{0\} \rightarrow S^{2}
$$

be a continuous spin configuration. The mapping $\mathbf{S}\left\lceil\Sigma: \Sigma \rightarrow S^{2}\right.$ is classified by its winding number $n \in \mathbb{Z}$. The number $n$ cannot change if $\Sigma$ is smoothly deformed, as long as the deformation does not go through the origin. We see thus that the topologically stable spin singularities are pointlike objects characterized by an integer winding number or topological charge. In the sequel the winding number will be referred to as charge. We adopt the name hedgehogs for the spin configurations that extremize the action $A$ and have charge concentrated at one point. This terminology is suggested by the name given by Polyakov [7] to similar configurations in field theory.

In so far as the influence on thermodynamics is concerned we expect our hedgehogs to be similar to the vortices in the two-dimensional $x y$-model. The action of a single vortex in the $2 d x y$-model is proportional to $\ln (V / a)$, where $V$ is the volume of the system and $a$ is the lattice spacing. A simple energy-entropy argument therefore shows that the vortices give an important contribution to the partition function at high temperature and in fact cause a phase transition [8-10]. In contrast to the $2 d x y$-model, where the existence of vortices is trivial, the existence of hedgehogs requires solving a system of nonlinear partial differential equations. Likewise the interaction of hedgehogs is more complex than the interaction of $x y$ vortices and cannot be calculated exactly.

The plan of the paper is as follows. In Sect. 2 we reduce the action (1.1) to a functional of a function of one variable by exploiting the anisotropic scale invariance of $A$. In Sect. 3 we use the results of Sect. 2 and functional analytic techniques to prove the existence of hedgehog configurations. Similar methods have been used to prove the existence of merons and monopoles in gauge theories $[11,12]$. Section 4 contains a proof of a lower bound for the action of an arbitrary spin configuration with a specified charge at one point. This section makes precise our statement about the logarithmic divergence of the action. In Sect. 5 we study the interaction of two equally and oppositely charged hedgehogs and derive upper and lower bounds for the interaction energy. These bounds suggest that the hedgehog-antihedgehog interaction is an anisotropic logarithmic function of the distance separating the two. This indicates that the hedgehogs can be regarded as a three-dimensional logarithmic gas, at least when they are dilute, in analogy with the vortices in the $2 d$ $x y$-model.

We would like to stress that it is doubtful whether the hedgehogs can cause a phase transition. Grest and Sak [13] have shown by a renormalization group argument that a system with the Hamiltonian (1.1) has a finite correlation length at all temperatures. The anisotropic logarithmic gas does not have a phase transition either, due to screening [3]. How the hedgehogs influence the thermodynamics will be the subject of a future investigation.

\section{A Cylindrically Symmetric Ansatz}

We begin by writing the action (1.1) in terms of unconstrained variables, the Euler angles of the spin vector: 


$$
\mathbf{S}=\left(S^{1}, S^{2}, S^{3}\right)=(\cos \phi \sin \theta, \sin \phi \sin \theta, \cos \theta) .
$$

After some algebra one finds

$$
\begin{aligned}
A(\mathbf{S})= & \int\left\{\left(\partial_{z} \theta\right)^{2}+\sin ^{2} \theta\left(\partial_{z} \phi\right)^{2}+(\nabla \theta)^{4}\right. \\
& +(\Delta \theta)^{2}+\sin ^{2} \theta\left[(\Delta \phi)^{2}+(\nabla \phi)^{4}+2(\nabla \phi)^{2}(\nabla \theta)^{2}\right] \\
& +4 \cos ^{2} \theta(\nabla \theta \cdot \nabla \phi)^{2} \\
& \left.+2 \sin \theta \cos \theta\left[2 \Delta \phi(\nabla \theta \cdot \nabla \phi)-\Delta \theta(\nabla \phi)^{2}\right]\right\} d^{3} x .
\end{aligned}
$$

We observe that the action (1.1) is invariant under the anisotropic scale transformation

$$
T_{\lambda}:(x, y, z) \rightarrow\left(\lambda x, \lambda y, \lambda^{2} z\right)
$$

where $\lambda$ is a constant; i.e. if we define $\mathbf{S}_{\lambda}(x, y, z)=\mathbf{S}\left(\lambda x, \lambda y, \lambda^{2} z\right)$, then $A\left(\mathbf{S}_{\lambda}\right)=A(\mathbf{S})$. Thus, if $\mathbf{S}$ describes a hedgehog at the origin, it is natural to assume that $\mathbf{S}$ only depends on the scale invariant quantities

$$
\left.\begin{array}{l}
t \equiv z\left(x^{2}+y^{2}\right)^{-1} \\
u \equiv y x^{-1}
\end{array}\right\}
$$

Now assume cylindrical symmetry, i.e.

$$
\phi(x, y, z)=\arctan u,
$$

and suppose $\theta$ is only a function of $t$. Then the action, which we now write as a functional of $\theta$, reduces to

$$
\begin{aligned}
A(\theta)= & \int\left\{\left(\partial_{z} \theta\right)^{2}+(\nabla \theta)^{4}+(\Delta \theta)^{2}-\sin (2 \theta) \Delta \theta r^{-2}\right. \\
& \left.+\sin ^{2} \theta\left[r^{-4}+2 r^{-2}(\nabla \theta)^{2}\right]\right\} d^{3} r .
\end{aligned}
$$

Integrating over the polar angle in the $x y$-plane, (2.6) becomes

$$
2 \pi \int_{0}^{\infty} d r r^{-1} B(\theta)
$$

where

$$
\begin{aligned}
B(\theta)= & \int_{-\infty}^{\infty}\left\{\left(\theta^{\prime}\right)^{2}+\left(2 t \theta^{\prime}\right)^{4}+\left[4\left(t \frac{d}{d t}\right)^{2} \theta\right]^{2}\right. \\
& \left.-4 \sin (2 \theta)\left(t \frac{d}{d t}\right)^{2} \theta+\sin ^{2} \theta\left[1+8\left(t \theta^{\prime}\right)^{2}\right]\right\} d t .
\end{aligned}
$$

The logarithmic divergence of the $r$-integral is manifest. We are reduced to studying the functional $B$.

For a hedgehog with charge $n$ the appropriate boundary conditions for $\theta$ are

$$
\left.\begin{array}{c}
\lim _{t \rightarrow-\infty} \theta(t)=0, \\
\lim _{t \rightarrow \infty} \theta(t)=n \pi .
\end{array}\right\}
$$


The boundary values (2.9) (modulo $\pi$ ) are forced by the cylindrical symmetry if we want to avoid a singularity along the $z$-axis.

\section{Existence of Hedgehogs}

In this section we prove that the functional $B$, subject to the boundary conditions (2.9), has a minimum on a suitable set of functions. The minimizing function is a solution of the variational equation of $B$. We begin by introducing some notation.

Let $C_{0}^{\infty}(\mathbb{R})$ denote the space of all infinitely differentiable real-valued functions on $\mathbb{R}$ that have compact support. Denote the $L^{p}$-norm by $\|\cdot\|_{p}$ and let $L$ be the differential operator $(x(d / d x))^{2}$. Let $X$ be the real Banach space obtained by completing $C_{0}^{\infty}(\mathbb{R})$ in the norm

$$
\|\theta\|=\left\|\theta^{\prime}\right\|_{2}+\left\|t \theta^{\prime}\right\|_{4}+\left\|\left(L-\frac{1}{4}\right) \theta\right\|_{2} .
$$

For each $n \in \mathbb{Z}$ we choose a $C^{\infty}$-function $\theta_{n}$ with the following property: $\theta_{n}(x)=0$ if $x<-1, \theta(x)=n \pi$ if $x>1$. We define a functional $B_{n}$ on $C_{0}^{\infty}(\mathbb{R})$ by $B_{n}(\theta)=B\left(\theta+\theta_{n}\right)$. Below we prove that $B_{n}$ extends to a well-defined, weakly lower semicontinuous (w.l.s.c.) and coercive functional on $X$. The main result of this section is the following.

Theorem 3.1. For any $n \in \mathbb{Z}$, the functional $B_{n}$ has a minimum on $X$.

We now establish a few preliminary Lemmas. Limits of integration are omitted when they are $\pm \infty$.

Lemma 3.2. Let $\theta \in X$. Then $\theta$ and $x \theta^{\prime}$ are bounded continuous functions and there are constants $k_{1}$ and $k_{2}$ such that

$$
\begin{aligned}
\|\theta\|_{\infty} & \leqq k_{1}\|\theta\|, \\
\left\|x \theta^{\prime}\right\|_{\infty} & \leqq k_{2}\|\theta\| .
\end{aligned}
$$

Proof. Since $C_{0}^{\infty}(\mathbb{R})$ is dense in $X$, we can assume $\theta \in C_{0}^{\infty}(\mathbb{R})$. By Hölder's inequality

$$
|\theta(x)|=\left|\int_{-\infty}^{x} \theta^{\prime} d t\right| \leqq 2\left\|t \theta^{\prime}\right\|_{4}\left[\int_{1}^{\infty} t^{-4 / 3} d t\right]^{3 / 4}+\sqrt{2}\left\|\theta^{\prime}\right\|_{2},
$$

which proves (3.2). Similarly,

$$
\begin{aligned}
\left(x \theta^{\prime}\right)^{2} & =2 \int_{-\infty}^{x}\left[t\left(\theta^{\prime}\right)^{2}+t^{2} \theta^{\prime} \theta^{\prime \prime}\right] d t \\
& =2 \int_{-\infty}^{x} \theta^{\prime}\left(L-\frac{1}{4}\right) \theta d t+\frac{1}{2} \theta^{2}(x) \\
& \leqq 2\left\|\theta^{\prime}\right\|_{2}\left\|\left(L-\frac{1}{4}\right) \theta\right\|_{2}+\frac{1}{2} k_{1}^{2}\|\theta\|^{2},
\end{aligned}
$$

which implies the desired result.

Lemma 3.3. If $\theta \in X$, then $\theta \in L^{p}(\mathbb{R})$ for any $p>2$ and there is a positive constant $C_{p}$ such that

$$
\|\theta\|_{p} \leqq C_{p}\|\theta\| .
$$


In other words: There is a continuous imbedding $i_{p}: X \rightarrow L^{p}(\mathbb{R})$ for any $p>2$.

Proof. Let $p>2$. It suffices to verify the inequality (3.6) for $\theta \in C_{0}^{\infty}(\mathbb{R})$. We have

$$
\int|\theta|^{p} d x=-p \int x \theta^{\prime} \operatorname{sgn} \theta|\theta|^{p-1} d x
$$

which implies

$$
\begin{aligned}
\frac{p-2}{2 p} \int|\theta|^{p} d x & \leqq\left\|x \theta^{\prime}+\frac{1}{2} \theta\right\|_{2}\left[\int|\theta|^{2(p-1)} d x\right]^{1 / 2} \\
& \leqq\left\|x \theta^{\prime}+\frac{1}{2} \theta\right\|_{2}\|\theta\|_{p}^{p / 2}\|\theta\|_{\infty}^{p-2 / 2}
\end{aligned}
$$

We now claim that

$$
\left\|x \theta^{\prime}+\frac{1}{2} \theta\right\|_{2} \leqq\left\|\left(L-\frac{1}{2}\right) \theta\right\|_{2},
$$

and the lemma then follows from (3.8) and (3.2). Integration by parts and Schwarz' inequality prove (3.9).

Lemma 3.4. The imbedding $i_{p}: X \rightarrow L^{p}(\mathbb{R})$ is compact for any $p>2$.

Proof. We only give the proof for $p=4$, since that is the only case we shall use. The proof for other values of $p$ is similar.

Let $V$ be the ball of radius $\rho$ in $X$. Then $V$ is precompact in $L^{4}(\mathbb{R})$ if and only if for all $\varepsilon>0$ there exists $\delta>0$ and a bounded subset $\Omega \subset \mathbb{R}$, such that for any $\theta \in V$ and $|h|$ $<\delta$,

$$
\int[\theta(x+h)-\theta(x)]^{4} d x<\varepsilon
$$

and

$$
\int_{\mathbb{R} \mid \Omega} \theta^{4} d x<\varepsilon
$$

see e.g. [14] p. 31. We begin by verifying (3.10). By the mean value theorem and Lemma 3.2 we have, assuming $|h| \leqq \frac{1}{2}$,

$$
\begin{aligned}
& \int[\theta(x+h)-\theta(x)]^{4} d x \\
& \quad \leq 2\left(2 h \rho k_{2}\right)^{4} \int_{1}^{\infty} x^{-4} d x+\left(2 k_{1} \rho\right)^{2} \int_{-1}^{1} d x\left[\int_{0}^{1} \frac{d}{d s} \theta(x+s h) d s\right]^{2} \\
& \quad=\frac{2}{5}\left(2 h \rho k_{2}\right)^{4}+\left(2 h k_{1} \rho\right)^{2} \int_{0}^{1} d t \int_{0}^{1} d s \int_{-1}^{1} d x \theta^{\prime}(x+s h) \theta^{\prime}(x+t h) \\
& \quad \leqq \frac{2}{5}\left(2 h \rho k_{2}\right)^{4}+\left(2 h k_{1} \rho\right)^{2}\left\|\theta^{\prime}\right\|_{2}^{2},
\end{aligned}
$$

which proves (3.10), provided $\delta$ is sufficiently small. To prove (3.11), let $\Omega=(-a, a)$ and take $a>1$. Then

$$
\int_{\mathbb{R} \backslash \Omega} \theta^{4} d x \leqq \sup _{|x|>a}|\theta(x)| \int_{\mathbb{R} \backslash \Omega}|\theta|^{3} d x
$$


and for $|x|>1$,

$$
|\theta(x)| \leqq k_{1}\|\theta\||x|^{-1 / 4}
$$

cf. Eq. (3.4). Hence, by Lemma 3.3, there is a constant $C$ such that

$$
\int_{\mathbb{R} \backslash \Omega} \theta^{4} d x \leqq C \rho^{4} a^{-1 / 4}
$$

for any $\theta \in V$. Taking a sufficiently large yields (3.11). This completes the proof.

We have now proven the imbedding theorems that we need. It is convenient to rewrite $B_{n}(\theta)$ as a sum of two functionals,

$$
B_{n}(\theta)=B(\theta)+R_{n}(\theta)
$$

where

$$
\begin{aligned}
R_{n}(\theta)= & \int_{-1}^{1}\left\{\left(\theta_{n}^{\prime}\right)^{2}+2 \theta_{n}^{\prime} \theta^{\prime}+\left[2 t\left(\theta^{\prime}+\theta_{n}^{\prime}\right)\right]^{4}-\left(2 t \theta^{\prime}\right)^{4}\right. \\
& +16\left(L \theta_{n}\right)^{2}+32\left(L \theta_{n}\right)(L \theta)-4 \sin \left(2 \theta_{n}+2 \theta\right) L \theta_{n} \\
& -4\left[\sin \left(2 \theta_{n}+2 \theta\right)-\sin (2 \theta)\right] L \theta+\sin ^{2}\left(\theta_{n}+\theta\right) \\
& -\sin ^{2} \theta+8 \sin ^{2}\left(\theta_{n}+\theta\right)\left[\left(t \theta_{n}^{\prime}\right)^{2}+2\left(t \theta_{n}^{\prime}\right)\left(t \theta^{\prime}\right)\right] \\
& \left.+8\left[\sin ^{2}\left(\theta_{n}+\theta\right)-\sin ^{2} \theta\right]\left(t \theta^{\prime}\right)^{2}\right\} d t .
\end{aligned}
$$

While the functional $R_{n}$ may look complicated, it is in fact much easier to deal with than $B$, because the integration is over a finite interval. We are now prepared to begin the analysis of $B_{n}$. Below, $c_{1}, c_{2}$ etc. will denote numerical constants whose values may change from one proof to another.

Proposition 3.5. The functional $B_{n}$ is well-defined and continuous on $X$ for any $n \in \mathbb{Z}$.

Proof. This follows easily from Lemmas 3.2 and 3.3.

Proposition 3.6. There are positive constants $a_{n}$ and $b_{n}$ such that

$$
B_{n}(\theta) \geqq a_{n}\left(\left\|\theta^{\prime}\right\|_{2}^{2}+\left\|t \theta^{\prime}\right\|_{4}^{4}+\left\|\left(L-\frac{1}{4}\right) \theta\right\|_{2}^{2}\right)-b_{n}
$$

for any $\theta \in X$.

Proof. From Lemma 3.2 it is clear that

$$
R_{n}(\theta) \geqq-c_{1}\left\|\theta^{\prime}\right\|_{2}-c_{2}\left\|t \theta^{\prime}\right\|_{4}^{3}-c_{3}\left\|\left(L-\frac{1}{4}\right) \theta\right\|_{2}-c_{4},
$$

where $c_{1}, c_{2}, c_{3}, c_{4}$ are positive and only depend on $n$. It therefore suffices to verify (3.18) with $B_{n}$ replaced by $B$. We have

$$
B(\theta) \geqq \int\left\{\left(\theta^{\prime}\right)^{2}+\left(2 t \theta^{\prime}\right)^{4}\right\} d t+\varepsilon \int\left\{(4 L \theta)^{2}-4 \sin (2 \theta) L \theta+\sin ^{2} \theta\right\} d t
$$

for any $\varepsilon \in(0,1)$. Using an integration by parts argument and Hölder's inequality we see that the $\varepsilon$-term in (3.20) is bounded from below by

$$
\begin{gathered}
\varepsilon\left\|\left(L-\frac{1}{4}\right) \theta\right\|_{2}^{2}-\varepsilon c_{5}\|\theta\|_{4}^{4}-\varepsilon c_{6}\|\theta\|_{4}^{2}\left\|t \theta^{\prime}\right\|_{4}^{2} \\
-\varepsilon c_{7}\|\theta\|_{4}^{3}\left\|t \theta^{\prime}\right\|_{4} .
\end{gathered}
$$


Choosing $\varepsilon$ sufficiently small, the desired result follows.

Remark. The reader may have wondered why we chose to use the norm $\left\|\left(L-\frac{1}{4}\right)(\cdot)\right\|_{2}$ rather than $\|L(\cdot)\|_{2}$, which might look more natural. The reason is that if we use $\|L(\cdot)\|_{2}$ the proof of coercivity breaks down, since all of the $L \theta$-term would be needed to control the negative term in $B$.

Proposition 3.7. The functional $B_{n}$ is weakly lower semicontinuous on $X$ for any $n \in \mathbb{Z}$.

Proof. We write $B_{n}(\theta)=A_{1}(\theta)+A_{2}(\theta)+A_{3}(\theta)$, where

$$
\begin{aligned}
& A_{1}(\theta) \equiv \int\left\{\left(\theta_{n}^{\prime}+\theta^{\prime}\right)^{2}+\left[2 t\left(\theta_{n}^{\prime}+\theta^{\prime}\right)\right]^{2}\right\} d t, \\
& A_{2}(\theta) \equiv 8 \int \sin ^{2}\left(\theta_{n}+\theta\right)\left(t \theta_{n}^{\prime}+t \theta^{\prime}\right)^{2} d t, \\
& A_{3}(\theta) \equiv \int\left\{\left[4 L\left(\theta_{n}+\theta\right)\right]^{2}-4 \sin \left(2 \theta_{n}+2 \theta\right) L\left(\theta_{n}+\theta\right)+\sin ^{2}\left(\theta_{n}+\theta\right)\right\} d t .
\end{aligned}
$$

We first observe that $A_{1}$ is convex in $\theta$ and thus w.l.s.c. Let now $\left\{\chi_{i}\right\} \subset X$ converge weakly to $\bar{\theta} \in X$. Then $\chi_{i}$ and $t \chi_{i}^{\prime}$ converge pointwise to $\bar{\theta}$ by Lemma 3.2. Hence, by Fatou's lemma, $A_{2}$ is w.l.s.c. It remains to consider $A_{3}$ which we can rewrite as

$$
\begin{aligned}
& \int\left\{\left[4\left(L-\frac{1}{4}\right) \theta\right]^{2}+\sin ^{2} \theta-\theta^{2}-[4 \sin (2 \theta)-8 \theta] L \theta\right\} d t \\
& +\int_{-1}^{1}\left\{32 L \theta_{n} L \theta+\sin ^{2}\left(\theta_{n}+\theta\right)-\sin ^{2} \theta-4 \sin \left(2 \theta_{n}+2 \theta\right) L \theta_{n}\right. \\
& \left.-4\left[\sin \left(2 \theta_{n}+2 \theta\right)-\sin (2 \theta)\right] L \theta\right\} d t+c_{1} .
\end{aligned}
$$

The integral from -1 to 1 above is weakly continuous in $\theta$ because weak convergence in $X$ implies uniform convergence on $[-1,1]$. The $\left(L-\frac{1}{4}\right)$-term in the first integral in (3.25) is convex and hence w.l.s.c. We complete the proof by showing that

$$
A_{4}(\theta) \equiv \int\left\{\sin ^{2} \theta-\theta^{2}-[4 \sin (2 \theta)-8 \theta] L \theta\right\} d t
$$

is weakly continuous. Let $\chi_{i}$ and $\bar{\theta}$ be as before. Then,

$$
\begin{aligned}
& \left|A_{4}(\bar{\theta})-A_{4}\left(\chi_{i}\right)\right| \leqq\left|\int\left(\sin ^{2} \bar{\theta}-\bar{\theta}^{2}-\sin ^{2} \chi_{i}+\chi_{i}^{2}\right) d t\right| \\
& \quad+\left|\int\left[4 \sin (2 \bar{\theta})-8 \bar{\theta}-4 \sin \left(2 \chi_{i}\right)+8 \chi_{i}\right] L \chi_{i} d t\right| \\
& \quad+\left|\int(4 \sin (2 \bar{\theta})-8 \bar{\theta}) L\left(\bar{\theta}-\chi_{i}\right) d t\right| .
\end{aligned}
$$

The last integral on the right of (3.27) is a linear continuous functional of $\bar{\theta}-\chi_{i}$ since it is bounded in absolute value by

$$
c_{2}\|\bar{\theta}\|_{6}^{3}\left\|\left(L-\frac{1}{4}\right)\left(\chi_{i}-\bar{\theta}\right)\right\|_{2}+c_{2}\|\bar{\theta}\|_{4}^{3}\left\|\chi_{i}-\bar{\theta}\right\|_{4} .
$$

The first two integrals on the right of (3.27) are bounded by

$$
c_{3} \int\left|\bar{\theta}-\chi_{i}\right|\left(|\bar{\theta}|^{3}+\left|\chi_{i}\right|^{3}\right) d t+c_{4} \int\left|\bar{\theta}-\chi_{i}\right|\left(\bar{\theta}^{2}+\chi_{i}^{2}\right)\left|L \chi_{i}\right| d t .
$$

Making use of Hölder's inequality, Lemma 3.3 and the fact that $\left\|\chi_{i}\right\|$ is bounded as $i \rightarrow \infty$, we see that there is a constant $c_{5}$ such that (3.29) is bounded from above by $c_{5}\left\|\bar{\theta}-\chi_{i}\right\|_{4}$. We conclude from Lemma 3.4 that $\lim \left\|\bar{\theta}-\chi_{i}\right\|_{4}=0$. This completes the proof. 
Proof of Theorem 3.1. It is easily checked that the Banach space $X$ is reflexive. Hence it follows from Propositions 3.6 and 3.7 by standard arguments that $B_{n}$ attains its infimum on $X$. For details see e.g. [15] p. 93.

\section{General Lower Bound for the Action}

In this section we derive a lower bound for the action in a finite volume containing no singularities. This estimate will be used in the next section to obtain a bound for the interaction energy of hedgehogs.

We leave the cylindrically symmetric ansatz considered in the last two sections and study an arbitrary field configuration $\mathbf{S}=\left(S^{1}, S^{2}, S^{3}\right)$, which is twice continuously differentiable in $\mathbb{R}^{3} \backslash\{0\}$ and has charge $n$ at 0 . Then, see e.g. [16] p. 568,

$$
n=(8 \pi)^{-1} \int_{\Sigma} \varepsilon_{a b c} S^{a} d S^{b} \wedge d S^{c}
$$

for any topological sphere $\Sigma$ enclosing 0 . For obvious reasons of convenience we work with cylinders and cylindrical surfaces.

Define

$$
\begin{aligned}
& \Omega_{R, L}=\left\{(x, y, z) \in \mathbb{R}^{3} \mid x^{2}+y^{2}<R,-\frac{1}{2} L<z<\frac{1}{2} L\right\}, \\
& D_{R, L}=\left\{(x, y, z) \in \mathbb{R}^{3} \mid x^{2}+y^{2}<R, z= \pm \frac{1}{2} L\right\}, \\
& H_{R, L}=\left\{(x, y, z) \in \mathbb{R}^{3} \mid x^{2}+y^{2}=R,-\frac{1}{2} L<z<\frac{1}{2} L\right\} .
\end{aligned}
$$

Then $\partial \Omega_{R, L}=D_{R, L} \cup H_{R, L}$. When there is no danger of confusion we shall omit the subscripts $R, L$. The main estimate is the following.

Theorem 4.1. Consider a cylinder $\Omega_{R, L}$ and let $d V$ be the volume form on $\partial \Omega_{R, L}$ inherited from $\mathbb{R}^{3}$. There are constants $m_{1}, m_{2}>0$, independent of $R$ and $L$, such that either

$$
\int_{D_{R, L}}\left\{\left(\partial_{z} \mathbf{S}\right)^{2}+(\Delta \mathbf{S})^{2}\right\} d V \geqq m_{1} n^{2} R^{-2}
$$

or

$$
\int_{H_{R, L}}\left\{\left(\partial_{z} \mathbf{S}\right)^{2}+(\Delta \mathbf{S})^{2}\right\} d V \geqq m_{2}|n|^{4 / 3}(R L)^{-1 / 3} .
$$

Before proving Theorem 4.1 we shall derive its consequence, which is more transparent. Let $L>l>0$ and define

$$
\Lambda(L, l)=\Omega_{L, L^{2}} \backslash \Omega_{l, l^{2}} .
$$

Theorem 4.2. There is a constant $m_{3}>0$, independent of $l$ and $L$, such that

$$
\int_{\Lambda(L, l)}\left\{\left(\partial_{z} \mathbf{S}\right)^{2}+(\Delta \mathbf{S})^{2}\right\} d^{3} x \geqq m_{3}|n|^{4 / 3} \ln \frac{L}{l} .
$$

Proof. (Assuming Theorem 4.1) Define

$$
A_{D}(\lambda)=\int_{D_{\lambda, \lambda^{2}}}\left\{\left(\partial_{z} \mathbf{S}\right)^{2}+(\Delta \mathbf{S})^{2}\right\} d V,
$$




$$
A_{H}(\lambda)=\int_{H_{\lambda, \lambda^{2}}}\left\{\left(\partial_{z} \mathbf{S}\right)^{2}+(\Delta \mathbf{S})^{2}\right\} d V
$$

Then

$$
\int_{\Lambda(L, l)}\left\{\left(\partial_{z} \mathbf{S}\right)^{2}+(\Delta \mathbf{S})^{2}\right\} d V=\int_{l}^{L}\left\{\lambda A_{D}(\lambda)+A_{H}(\lambda)\right\} d \lambda,
$$

and using now Theorem 4.1, (4.11) is larger than

$$
m_{3}|n|^{4 / 3} \ln \frac{L}{l}
$$

with $m_{3}=\min \left\{m_{1}, m_{2}\right\}$.

Proof of Theorem 4.1. Let $r, z, \chi$ be cylindrical coordinates in $\mathbb{R}^{3}$. By formula (4.1) we have

$$
\begin{aligned}
8 \pi n= & \varepsilon_{a b c}\left\{\int_{D} S^{a}\left(\frac{\partial S^{b}}{\partial \chi} \frac{\partial S^{c}}{\partial r}-\frac{\partial S^{b}}{\partial r} \frac{\partial S^{c}}{\partial \chi}\right) r^{-1} d V\right. \\
& \left.+\int_{H} S^{a}\left(\frac{\partial S^{b}}{\partial z} \frac{\partial S^{c}}{\partial \chi}-\frac{\partial S^{b}}{\partial \chi} \frac{\partial S^{c}}{\partial z}\right) R^{-1} d V\right\} .
\end{aligned}
$$

If $\mathbf{u}$ is a three-dimensional vector, let $|\mathbf{u}|$ denote its Euclidean length. Since $a, b, c$ run from 1 to 3 it follows that

$$
\begin{aligned}
8 \pi n & \leqq 12 \int_{D}\left|\frac{\partial \mathbf{S}}{\partial r}\right|\left|\frac{\partial \mathbf{S}}{r \partial \chi}\right| d V+12 \int_{H}\left|\frac{\partial \mathbf{S}}{\partial z}\right|\left|\frac{\partial \mathbf{S}}{R \partial \chi}\right| d V \\
& \leqq 12 \int_{D}(\nabla \mathbf{S})^{2} d V+12\left\{\int_{H}\left(\frac{\partial \mathbf{S}}{\partial z}\right)^{2} d V \int_{H}(\nabla \mathbf{S})^{2} d V\right\}^{1 / 2},
\end{aligned}
$$

using the Schwarz inequality. Since $\mathbf{S} \cdot \mathbf{S}=1$, we have

$$
\mathbf{S} \cdot \Delta \mathbf{S}+\nabla \mathbf{S} \cdot \nabla \mathbf{S}=0
$$

Hence,

$$
\int_{D}(\nabla \mathbf{S})^{2} d V \leqq \int_{D}|\Delta \mathbf{S}| d V \leqq(2 \pi)^{1 / 2} R\left[\int_{D}(\Delta \mathbf{S})^{2} d V\right]^{1 / 2},
$$

and similarly

$$
\int_{H}(\nabla \mathbf{S})^{2} d V \leqq(2 \pi R L)^{1 / 2}\left[\int_{I I}(\Delta \mathbf{S})^{2} d V\right]^{1 / 2} .
$$

From Eq. (4.14), (4.16) and (4.17) we obtain

$$
\frac{2}{3} \pi n \leqq(2 \pi)^{1 / 2} R g+(2 \pi R L)^{1 / 4}\left[\int_{H}(\Delta \mathbf{S})^{2} d V\right]^{1 / 4}\left[\int_{H}\left(\frac{\partial \mathbf{S}}{\partial z}\right)^{2} d V\right]^{1 / 2}
$$

where

$$
g \equiv\left(\int_{D}(\Delta \mathbf{S})^{2} d V\right)^{1 / 2}
$$


Now we consider two cases.

Case 1 . $R g \geqq(n / 3)(\pi / 2)^{1 / 2}$. Then $g^{2} \geqq\left(n^{2} / 18\right) \pi R^{-2}$, which implies (4.5).

Case 2. $R g<(n / 3)(\pi / 2)^{1 / 2}$. Then,

$$
\frac{\pi^{2}}{9} n^{2} \leqq(2 \pi R L)^{1 / 2} e f^{2}
$$

where

$$
e \equiv\left(\int_{H}(\Delta \mathbf{S})^{2} d V\right)^{1 / 2}
$$

and

$$
f \equiv\left(\int_{H}\left(\frac{\partial \mathbf{S}}{\partial z}\right)^{2} d V\right)^{1 / 2}
$$

Hence,

$$
e^{2}+f^{2} \geqq e^{2}+\frac{\alpha}{e}
$$

where $\alpha \equiv\left(\pi^{2} / 9\right) \mathrm{n}^{2}(2 \pi R L)^{-1 / 2}$, which implies (4.6).

\section{The Interaction of Hedgehogs}

In this section we study the energy of a spin configuration with charge 1 at the origin and change -1 at another point $\mathbf{r}$. Our estimates give upper and lower bounds for the interaction energy of a hedgehog and an antihedgehog.

Since the action density is invariant under the generalized scale transformations (2.3) it is natural to try to find an "inversion" that leaves the action density invariant and can be used, combined with a translation, to construct a hedgehogantihedgehog configuration from the one hedgehog configuration. This method works nicely for merons [17], where one has conformal invariance. However, an explicit calculation shows that there is no mapping from the one point compactification of $\mathbb{R}^{3}$ onto itself that interchanges 0 and $\infty$ and leaves the action density invariant.

Let $\mathscr{S}(\mathbf{r})$ be the class of all spin configurations $\mathbf{S}$ with charge 1 at 0 and -1 at $\mathbf{r}=(x, y, z)$ that are twice continuously differentiable except at 0 and $\mathbf{r}$. The hedgehog-antihedgehog configuration with the specified charges should belong to the class $\mathscr{S}(\mathbf{r})$. We know from the last section that the action in small cylinders containing the singularities is logarithmically divergent. We therefore introduce a short distance cutoff as follows. Denote the unit cylinder $\Omega_{1,1}$ by $\Omega$ and denote by $\Lambda(\mathbf{r})$ the set obtained by removing the cylinders $\Omega$ and $\mathbf{r}+\Omega$ from $\mathbb{R}^{3}$. Let

$$
\Gamma=\left\{\mathbf{r} \in \mathbb{R}^{3} \mid \operatorname{dist}(\Omega, \mathbf{r}+\Omega)>\frac{1}{2}\right\} .
$$

We shall derive upper and lower bounds for the quantity

$$
E(\mathbf{S}) \equiv \int_{\Lambda(\mathbf{r})}\left\{\left(\partial_{z} \mathbf{S}\right)^{2}+(\Delta \mathbf{S})^{2}\right\} d^{3} x
$$


with $\mathbf{S} \in \mathscr{S}(\mathbf{r})$ and $\mathbf{r} \in \Gamma$. The above cutoff is chosen solely for notational convenience. One can choose the cylinders $\Omega$ of a different size and then obtain a different range for $\mathbf{r}$.

Choose $\mathbf{r} \in \Gamma$ and let

$$
d=\max \left\{|z|^{1 / 2}, \frac{1}{2}\left(x^{2}+y^{2}\right)^{1 / 2}\right\} .
$$

Then the intersection of the cylinders $\Omega_{d, d^{2}}$ and $\mathbf{r}+\Omega_{d, d^{2}}$ is empty. From Theorem 4.2 we conclude

$$
E(\mathbf{S}) \geqq 2 m_{3} \ln d,
$$

which implies the first half of the following theorem.

Theorem 5.1. There are positive constants $E_{1}, E_{2}$ such that for any $\mathbf{r} \in \Gamma$

$$
E(\mathbf{S}) \geqq E_{1} \ln |\mathbf{r}|
$$

for any $\mathbf{S} \in \mathscr{S}(\mathbf{r})$, and there exists $\mathbf{S} \in \mathscr{S}(\mathbf{r})$ such that

$$
E(\mathbf{S}) \leqq E_{2} \ln |\mathbf{r}| .
$$

Proof. Consider those $\mathbf{r} \in \Gamma$ that belong to a fixed parabola $P_{t, u}=\{(x, y, z) \mid y / x=u$, $\left.z / x^{2}+y^{2}=t\right\}$. Let $\mathbf{r}_{0}$ be one of the two points in $\Gamma \cap P_{t, u}$ closest to 0 . Choose a field configuration $\mathbf{S}_{0} \in \mathscr{S}\left(\mathbf{r}_{0}\right)$ with the following properties:

i) In $\Omega, \mathbf{S}_{0}$ is equal to a hedgehog configuration with charge 1 at 0 .

ii) In $\mathbf{r}_{0}+\Omega, \mathbf{S}_{0}$ is equal to a hedgehog configuration with charge -1 at $\mathbf{r}_{0}$.

iii) $\mathbf{S}_{0}$ is constant outside a bounded region.

The existence of a field configuration $\mathbf{S}_{0}$ satisfying (i-iii) is clear from standard extension theorems. Furthermore, it is not hard to check that one can choose the $\mathbf{S}_{0}$ 's such that

$$
\sup _{\substack{-\infty \leq t \leq \infty \\-\infty \leq u \leq \infty}} E\left(\mathbf{S}_{0}\right) \equiv E<\infty .
$$

For any $\lambda \geqq 1$, define $\mathbf{S}_{0}^{\lambda}(\mathbf{r})=\mathbf{S}_{0}\left(T_{\lambda^{-1}} \mathbf{r}\right)$, where $T_{\lambda}$ is the scale transformation (2.3). Then $\mathbf{S}_{0}^{\lambda} \in \mathscr{S}\left(T_{\lambda} \mathbf{r}_{0}\right)$ and

$$
\begin{aligned}
& E\left(\mathbf{S}_{0}^{\lambda}\right)=\int_{A\left(T_{\left.\lambda \boldsymbol{r}_{0}\right)}\right.}\left\{\left(\partial_{z} \mathbf{S}_{0}^{\lambda}\right)^{2}+\left(\Delta \mathbf{S}_{0}^{\lambda}\right)^{2}\right\} d^{3} x \\
& =E\left(\mathbf{S}_{0}\right)+\int_{\Xi}\left\{\left(\partial_{z} \mathbf{S}_{0}\right)^{2}+\left(\Delta \mathbf{S}_{0}\right)^{2}\right\} d^{3} x,
\end{aligned}
$$

where $\Xi=T_{\lambda^{-1}}\left(\Lambda\left(T_{\lambda} \mathbf{r}_{0}\right)\right) \backslash \Lambda\left(\mathbf{r}_{0}\right)$. Using (i) and (ii) we see that the integral over $\Xi$ is bounded from above by $4 \pi b_{1} \ln \lambda$, where $b_{1}$ is the infimum of the functional $B_{1}$ on $X$. In view of (5.7) the inequality (5.6) follows easily.

We make two final comments. An inspection of the proof of the bounds (5.5) and (5.6) indicates that the interaction energy increases twice as fast in the $x y$ direction as it does in the $z$-direction.

It is not possible to use the methods above without some modifications to estimate a priori the interaction energy of two identically charged hedgehogs, 
because the action integral will diverge at infinity. In order to remove this divergence one would have to introduce a third charge to ensure the neutrality of the system.

Acknowledgement. I would like to thank John Hertz for suggesting the study of the hedgehogs and reading the manuscript.

\section{References}

1. Hornreich, R. M., Luban, M., Shtrikman, S.: Critical behavior at the onset of k-space instability on the $\lambda$ line. Phys. Rev. Lett. 35, 1678-1681 (1975)

2. Hornreich, R. M.: Renormalization-group analysis of critical modes at the ferromagnetic Lifshitz point in crystalline systems. Phys. Rev. B19, 5914-5920 (1979)

3. Grinstein, G.: Anisotropic Sine-Gordon model and infinite-order phase transitions in three dimensions. Phys. Rev. B23, 4615-4630 (1981); Spin-wave theory for the biaxial $(m=2)$ Litshitz point problem in three dimensions. J. Phys. A13, L201-L205 (1980)

4. Grinstein, G., Pelcovits, R. A.: Anharmonic effects in bulk smectic liquid crystals and other "onedimensional" solids. Phys. Rev. Lett. 47, 856-859 (1981)

5. Nelson, D. R., Toner, J.: Bond-orientational order, dislocation loops, and melting of solids and smectic-A liquid crystals. Phys. Rev. B24, 363-387 (1981)

6. Toner, J.: Renormalization group treatment of the dislocation loop model of the smectic A-nematic transition Phys. Rev. B26, 462-465 (1982)

7. Polyakov, A. M.: Particle spectrum in quantum field theory. Sov. Phys. JETP Lett. 20, 194-195 (1974); Isomeric states of quantum fields. Sov. Phys. JETP 41, 988-995 (1976)

8. Kosterlitz, J. M., Thouless, D. J.: Ordering, metastability and phase transitions in two-dimensional systems. J. Phys. C6, 1181-1203 (1973)

9. Kosterlitz, J. M.: The critical properties of the two-dimensional $x y$ model. J. Phys. C7, 1046-1060 (1974)

10. Fröhlich, J., Spencer, T.: The Kosterlitz-Thouless transition in two-dimensional Abelian spin systems and the Coulomb gas. Commun. Math. Phys. 81, 527-602 (1981)

11. Jonsson, T., McBryan, O., Zirilli, F., Hubbard, J.: An existence theorem for multimeron solutions to classical Yang-Mills field equations. Commun. Math. Phys. 68, 259-273 (1979)

12. Jaffe, A., Taubes, C.: Vortices and monopoles. Boston, Basel, Stuttgart: Birkhäuser 1980

13. Grest, G. S., Sak, J.: Low-temperature renormalization group for the Lifshitz point. Phys. Rev. B17, 3607-3610 (1978)

14. Adams, R.: Sobolev spaces. New York, San Francisco, London: Academic Press 1975

15. Vainberg, M.: Variational method and the method of monotone operators in the theory of nonlinear equations. New York: Wiley 1973

16. Felsager, B.: Geometry, particles and fields. Odense: Odense University Press 1981

17. De Alfaro, V., Fubini, S., Furlan, G.: A new classical solution of the Yang-Mills field equations. Phys. Lett. 65B, 163-167 (1976)

Communicated by J. Fröhlich

Received September 16, 1982; in revised form February 5, 1983 